\title{
Association between human papillomavirus and EGFR mutations in advanced lung adenocarcinoma
}

\author{
MING LI ${ }^{1,2}$, FANG DENG ${ }^{1,2}$, LI-TING QIAN ${ }^{2}$, SHUI-PING MENG ${ }^{3}$, YANG ZHANG ${ }^{2}$, \\ WU-LIN SHAN $^{2}$, XIAO-LEI ZHANG ${ }^{2}$ and BAO-LONG WANG ${ }^{1}$ \\ ${ }^{1}$ Department of Clinical Laboratory, Affiliated Provincial Hospital of Anhui Medical University, Hefei, \\ Anhui 230001; ${ }^{2}$ Department of Clinical Laboratory; ${ }^{3}$ Department of Respiratory Oncology, \\ Anhui Provincial Cancer Hospital, Hefei, Anhui 230031, P.R. China
}

Received April 2, 2015; Accepted June 2, 2016

DOI: $10.3892 / \mathrm{ol} .2016 .4847$

\begin{abstract}
Previous studies have demonstrated an association between human papillomavirus (HPV) and mutations in the epidermal growth factor receptor $(E G F R)$ gene in lung cancer patients; however, few studies have investigated this association in advanced lung adenocarcinoma patients undergoing gefitinib treatment. The present study investigated the association between HPV and EGFR mutations in advanced lung adenocarcinoma patients. A total of 95 advanced lung adenocarcinoma patients were enrolled in the study. The HPV infection status and presence of EGFR mutations in tumor tissue was evaluated. Patient clinical characteristics were also determined and compared with HPV infection and EGFR mutation status to analyze their impact on progression-free survival. HPV DNA was identified in 27/95 (28.4\%) lung adenocarcinoma tumors and was most common in patients with lymph node metastasis $(\mathrm{P}=0.016)$. A total of 44/95 (46.3\%) cases exhibited EGFR mutations, which were predominantly observed in female patients and non-smokers. The presence of HPV DNA was significantly associated with EGFR mutations $(\mathrm{P}=0.012)$ and multivariate analysis also revealed that HPV DNA was significantly associated with EGFR mutations (odds ratio=3.971) in advanced lung adenocarcinoma. Patients with both HPV infections and EGFR mutations exhibit a marked decrease in the risk of lung cancer progression when compared with those without HPV infection or EGFR mutations (adjusted $\mathrm{HR}=0.640 ; 95 \%$ confidence interval: 0.488-0.840; $\mathrm{P}=0.001)$. HPV infection was significantly associated with EGFR mutations in advanced lung adenocarcinoma patients. Furthermore, patients with HPV infections
\end{abstract}

Correspondence to: Professor Bao-Long Wang, Department of Clinical Laboratory, Affiliated Provincial Hospital of Anhui Medical University, 17 Lujiang Road, Hefei, Anhui 230001, P.R. China

E-mail: 18919685630@163.com

Key words: human papillomavirus, epidermal growth factor receptor, mutation, lung adenocarcinoma, survival exhibited the longest progression-free survival times, which may be due to good response to tyrosine kinase inhibitor- or platinum-based-adjuvant therapy in these patients. Patients with EGFR mutations exhibited a better prognosis when compared with those exhibiting wild-type EGFR, regardless of HPV status.

\section{Introduction}

Lung cancer is the leading cause of cancer mortality, worldwide (1). Although cigarette smoking is the primary risk factor for lung cancer, the epidemiology of lung cancer remains partially unresolved, as the majority of cigarette smokers do not develop lung tumors. Furthermore, lung cancer is currently the seventh leading cause of cancerrelated mortality in non-smokers (2). Human papillomavirus (HPV) infection has been postulated as a possible risk factor for the disease (3).

Recent studies have identified differences in the prevalence of HPV infection in lung cancer between different ethnicities and geographical variations $(4,5)$. A lack of consensus with regard to detection methods, as well as methodological flaws may have been responsible for the large discrepancies reported, particularly between studies reporting conflicting results with regard to the prevalence of HPV in areas within the same geographical locations (6). Due to the variability in HPV prevalence reported, the involvement of HPV in lung cancer, specifically in non-small cell lung cancer (NSCLC), which accounts for $80 \%$ of all lung cancer cases, remains to be elucidated $(7,8)$.

Previous studies in Taiwanese and Japanese populations have reported that female non-smokers with lung adenocarcinoma exhibit an elevated incidence of HPV infections (9) and observed that lung cancer patients with HPV infections were responsive to gefitinib treatment (10). Recently, two studies demonstrated that mutations in the epidermal growth factor receptor $(E G F R)$ gene were associated with the presence of HPV infection in Taiwanese and Japanese patients $(11,12)$. However, these studies did not evaluate the response rate to gefitinib as all subjects were diagnosed with primary lung cancer and subsequently underwent surgery rather than gefitinib administration. 
In the present study, 95 patients with advanced lung adenocarcinoma were enrolled to retrospectively investigate the association between HPV status, EGFR mutations and clinical characteristics, as well as their impact on progression-free survival (PFS).

\section{Materials and methods}

Clinical specimens. A total of 95 paraffin-embedded tissue samples were obtained from advanced lung adenocarcinoma patients (stage III-IV) (13) prior to adjuvant therapy at the Department of Respiratory Oncology, Anhui Provincial Hospital (Hefei, China) between August 2011 and December 2013. Patients with complete clinicopathological and follow-up data were included. The tissues included 20 surgical specimens, 18 lung biopsy specimens, 18 bronchoscopic biopsy specimens, 23 pleural effusion specimens, 13 lymph node biopsy specimens and 3 bone biopsy specimens. All patients provided written informed consent was provided and the study was approved by the Institutional Review Board of Anhui Provincial Hospital.

Clinical characteristics including patient age, gender, smoking history, histological differentiation, lymph node metastasis, distant metastasis and treatment type [tyrosine kinase inhibitors (TKIs) (gefitinib or erlotinib) and platinum-based chemotherapy (cisplatin or carboplatin)] were collected for subsequent analyses. PFS was defined as the time from treatment to recurrence or the date of censorship (the last date of follow-up).

DNA extraction. DNA was deparaffinized and extracted from tissue samples using the QIAamp DNA FFPE Tissue kit (Qiagen, Hilden, Germany) according to the manufacturer's instructions. Briefly, 5-6 formalin-fixed, paraffin-embedded $8-\mu \mathrm{m}$ tissue sections were soaked in xylene and vortexed vigorously. A tissue pellet was obtained and purified according to the manufacturer's instructions. DNA samples were quantified spectrophotometrically and normalized aliquots were obtained for each sample.

HPV DNA detection. HPV testing of lung cancer samples was performed using polymerase chain reaction (PCR) amplification of a fragment of the HPV Ll gene. The presence of HPV infection was determined using a Tellgenplex ${ }^{\mathrm{TM}}$ HPV DNA Test kit (Tellgen Life Science Co., Ltd., Shanghai, China) using the Luminex ${ }^{\circledR}$ technique, which enables the detection of 26 HPV genotypes, including those of 19 high-risk HPV types $(16,18,26,31,33,35,39,45,51,52,53,55,56,58,59,66,68,82$ and 83 ) and 7 low-risk HPV types $(6,11,40,42,44,61$ and 73$)$. The experiments were performed in a Bio-Plex ${ }^{\circledR} 200$ system (Bio-Rad Laboratories, Hercules, CA, USA) according to the manufacturer's instructions. Briefly, $2 \mu \mathrm{g}$ extracted DNA was amplified by PCR under the following conditions: 5 cycles of $95^{\circ} \mathrm{C}$ for $30 \mathrm{sec}, 58^{\circ} \mathrm{C}$ for $30 \mathrm{sec}$ and $72^{\circ} \mathrm{C}$ for $30 \mathrm{sec}$ followed by 35 cycles of $95^{\circ} \mathrm{C}$ for $30 \mathrm{sec}, 55^{\circ} \mathrm{C}$ for $30 \mathrm{sec}$ and $72^{\circ} \mathrm{C}$ for $30 \mathrm{sec}$. PCR products were subjected to rapid hybridization and the data were analyzed using the software provided by the manufacturer (Tellgen Software, version IS2.3; Tellgen Life Science Co., Ltd.) (14).

Mutations in EGFR exons 18-21 were detected using previously described methods (15).
Statistical analysis. Statistical analyses were performed using SPSS 16.0 statistical software (SPSS, Inc., Chicago, IL, USA). $\chi^{2}$ tests were used to compare the associations between HPV status, EGFR mutation status and clinical characteristics. To investigate the combined effect of HPV infection and $E G F R$ mutation on PFS, four subgroups were determined $\left(\mathrm{HPV}^{+} /\right.$mutant $^{+}, \mathrm{HPV}^{+} /$mutant ${ }^{-}, \mathrm{HPV}^{-} /$mutant ${ }^{+}$, and $\mathrm{HPV}^{-} /$mutant ${ }^{-}$). The survival curves were estimated by Kaplan-Meier methods and the differences in survival distributions were compared using the log-rank test. To evaluate the mortality risk, the hazard ratio (HR) and corresponding $95 \%$ confidence interval (CI) were estimated using Cox proportional hazards models to identify potential prognostic factors. All statistical tests were two-sided and $\mathrm{P}<0.05$ was considered to indicate statistically significant differences.

\section{Results}

High-risk HPV infection in advanced lung adenocarcinoma patients. Of the 95 DNA samples tested, 27 (28.4\%) were positive for high-risk HPV. The most common high-risk HPV types identified in lung adenocarcinoma patients were HPV16 and HPV18; however, other genotypes including HPV33 and 58 were also detected.

Association between EGFR mutation and HPV infection. HPV infection was associated with lymph node metastasis $(\mathrm{P}=0.016)$ (Table I). A total of 44/95 cases (46.3\%) exhibited EGFR mutations (in exons 19/21). EGFR mutations were more common in females $(\mathrm{P}<0.006)$ and never-smokers $(\mathrm{P}<0.019)$ when compared with males and ever-smokers, respectively. The presence of HPV DNA was significantly associated with EGFR mutations $(\mathrm{P}=0.012)$ (Table I). Multivariate analysis also revealed that HPV DNA was significantly associated with EGFR mutations (odds ratio=3.971) in advanced lung adenocarcinoma (Table II).

Impact of HPV infection and EGFR mutations on PFS. To determine the clinical significance of HPV infection status and EGFR mutations in advanced lung adenocarcinoma, the association between HPV infection, EGFR mutations and PFS was investigated in the present study. The Kaplan-Meier survival curve estimates are shown in Fig. 1. Patients with HPV infections exhibited a longer median PFS time than those without (13 vs. 9 months; $\mathrm{P}=0.013$ ).

To evaluate the clinicopathological characteristics of patients and the impact of such on PFS in advanced lung adenocarcinoma, the HR and corresponding 95\% CI were estimated using the Cox proportional hazards analysis, which was adjusted for smoking (never vs. ever), lymph node metastasis (yes vs. no), EGFR (mutated vs. wild-type) and HPV infection (positive vs. negative). The presence of EGFR mutations and lymph node metastasis were independent prognostic factors for survival, however, this trend was not observed in patients with HPV infection (Table III).

For advanced lung adenocarcinoma, treatment modality is one of the most important determinants of clinical outcome, thus, the PFS of patients treated with TKIs and platinum-based chemotherapy was analyzed using the Cox proportional hazards model. No significant difference in survival was 
Table I. Clinicopathological characteristics, HPV infection status and EGFR mutation status of advanced lung adenocarcinoma patients.

\begin{tabular}{|c|c|c|c|c|c|c|}
\hline \multirow[b]{2}{*}{ Characteristics } & \multicolumn{2}{|c|}{$\mathrm{HPV}$} & \multirow[b]{2}{*}{ P-value } & \multicolumn{2}{|c|}{ EGFR } & \multirow[b]{2}{*}{ P-value } \\
\hline & Positive, $\mathrm{n}$ & Negative, $\mathrm{n}$ & & Mutated, n & Wild-type, $\mathrm{n}$ & \\
\hline Age, years & & & 0.415 & & & 0.448 \\
\hline$<64$ & 16 & 34 & & 25 & 25 & \\
\hline$\geq 64$ & 11 & 34 & & 19 & 26 & \\
\hline Gender & & & 0.255 & & & 0.006 \\
\hline Male & 12 & 39 & & 17 & 34 & \\
\hline Female & 15 & 29 & & 27 & 17 & \\
\hline Smoking status & & & 0.078 & & & 0.019 \\
\hline Never & 20 & 37 & & 32 & 25 & \\
\hline Ever & 7 & 31 & & 12 & 26 & \\
\hline Histological differentiation & & & 0.109 & & & 0.110 \\
\hline Well & 4 & 21 & & 10 & 15 & \\
\hline Poor & 23 & 47 & & 29 & 41 & \\
\hline Lymph node metastasis & & & 0.016 & & & 0.801 \\
\hline Yes & 11 & 46 & & 27 & 30 & \\
\hline No & 16 & 22 & & 17 & 21 & \\
\hline Distant metastasis & & & 0.255 & & & 0.326 \\
\hline Yes & 12 & 39 & & 26 & 25 & \\
\hline No & 15 & 29 & & 18 & 26 & \\
\hline EGFR mutations & & & 0.012 & & & \\
\hline Mutated & 18 & 26 & & & & \\
\hline Wild-type & 9 & 42 & & & & \\
\hline
\end{tabular}

HPV, human papillomavirus; EGFR, epidermal growth factor receptor.

Table II. Multivariate analysis of the association between human papillomavirus infection status and the clinicopathological characteristics of patients with advanced lung adenocarcinoma.

\begin{tabular}{lccc}
\hline & & Multivariate logistic regression \\
\cline { 2 - 4 } Variables & Odds ratio & 95\% confidence interval & $0.252-3.358$ \\
\hline Gender (male vs. female) & 0.920 & $0.279-2.179$ & 0.899 \\
Age, years (<64 vs. $\geq 64)$ & 0.779 & $0.165-2.579$ & 0.635 \\
Smoking status (never vs. ever) & 0.652 & $0.087-1.192$ & 0.542 \\
Histological differentiation (well vs. poor) & 0.321 & $0.125-1.039$ & 0.090 \\
Lymph node metastasis (yes vs. no) & 0.361 & $0.220-1.892$ & 0.059 \\
Distant metastasis (yes vs. no) & 0.645 & $1.364-11.562$ & 0.424 \\
EGFR (mutated vs. wild-type) & 3.971 & 0.011 \\
\hline
\end{tabular}

EGFR, epidermal growth factor receptor.

identified between patients with HPV infections and those without in the subgroups receiving TKI and platinum-based chemotherapy (Table IV). The results indicated that the longest PFS times, which were observed in patients with positive HPV infection status, may have been due to a good response to TKIor platinum-based-adjuvant therapy.
HPV infection and EGFR mutation exhibit a synergistic effect on PFS time. Multivariate analysis demonstrated that HPV DNA was significantly associated with EGFR mutations in lung adenocarcinoma and thus, the combined effects of these two biomarkers on PFS was evaluated, which revealed that they exhibited a synergistic benefit on PFS time. Patients with 
Table III. Univariate and multivariate analyses of progression-free survival.

\begin{tabular}{|c|c|c|c|c|c|c|}
\hline \multirow[b]{2}{*}{ Variables } & \multicolumn{2}{|c|}{ Univariate } & \multirow[b]{2}{*}{ P-value } & \multicolumn{2}{|c|}{ Multivariate } & \multirow[b]{2}{*}{ P-value } \\
\hline & HR & $95 \% \mathrm{CI}$ & & HR & $95 \% \mathrm{CI}$ & \\
\hline Gender (male vs. female) & 0.888 & $0.569-1.387$ & 0.602 & & & \\
\hline Age, years $(<64$ vs. $\geq 64)$ & 1.034 & $0.665-1.608$ & 0.881 & & & \\
\hline Smoking status (never vs. ever) & 1.709 & $1.086-2.690$ & 0.020 & 1.174 & $0.738-1.866$ & 0.498 \\
\hline Histological differentiation (well vs. poor) & 0.829 & $0.494-1.391$ & 0.478 & & & \\
\hline Lymph node metastasis (yes vs. no) & 2.756 & $1.618-4.696$ & $<0.001$ & 2.750 & $1.566-4.829$ & $<0.001$ \\
\hline Distant metastasis (yes vs. no) & 1.441 & $0.922-2.254$ & 0.109 & & & \\
\hline EGFR status (mutated vs. wild-type) & 0.229 & $0.134-0.392$ & $<0.001$ & 0.229 & $0.132-0.397$ & $<0.001$ \\
\hline HPV infection (positive vs. negative) & 0.556 & $0.337-0.915$ & 0.021 & 0.893 & $0.518-1.539$ & 0.683 \\
\hline
\end{tabular}

CI, confidence interval; HR, hazard ratio; EGFR, epidermal growth factor receptor; HPV, human papillomavirus.

Table IV. Subgroup analyses for PFS stratified by treatments.

\begin{tabular}{llccc}
\hline \multirow{2}{*}{ Treatment } & $\begin{array}{c}\text { HPV } \\
\text { status }\end{array}$ & $\begin{array}{c}\text { Median PFS } \\
\text { time, months }\end{array}$ & $\begin{array}{c}\text { HR } \\
(95 \% \mathrm{CI})\end{array}$ & P-value \\
\hline TKI & Positive & 16 & 0.689 & 0.304 \\
& Negative & 14 & $(0.338-1.402)$ & \\
\multirow{2}{*}{ Platinum } & Positive & 10 & 0.600 & 0.170 \\
& Negative & 7 & $(0.290-1.245)$ & \\
\hline
\end{tabular}

PFS, progression-free survival; HPV, human papillomavirus; HR, hazard ratio; TKI, tyrosine kinase inhibitor; CI, confidence interval.

both HPV infection and EGFR mutations exhibited a significantly reduced risk when compared with those exhibiting neither biomarker (adjusted HR=0.640; 95\% CI: 0.488-0.840; $\mathrm{P}=0.001$; Table V). The PFS curves are shown in Fig. 2. In addition, the results demonstrated that patients with EGFR mutations exhibited a better prognosis compared with those exhibiting wild-type EGFR, regardless of HPV status.

\section{Discussion}

In the present study, 27/95 (28.4\%) advanced lung adenocarcinoma patients were positive for high-risk HPV infection. Multivariate analysis revealed a significant association between HPV infection and EGFR mutations. Subgroup analyses for PFS stratified by treatments indicated that patients with HPV infections exhibited the longest PFS times in both the TKI and platinum-based-adjuvant treatment groups, which may be due to patients exhibiting a good response to TKI- or platinum-based-adjuvant therapy. Patients with both $E G F R$ mutations and HPV infections $\left(\mathrm{HPV}^{+} /\right.$mutant $\left.{ }^{+}\right)$exhibited the longest survival time when compared with the other subgroups

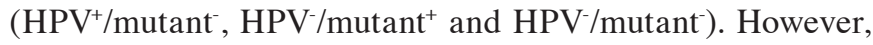
patients with EGFR mutations exhibit a better prognosis compared with those exhibiting wild-type $E G F R$, regardless of HPV status.

The reported frequency of HPV infection in lung cancer patients varies worldwide. A comprehensive literature review

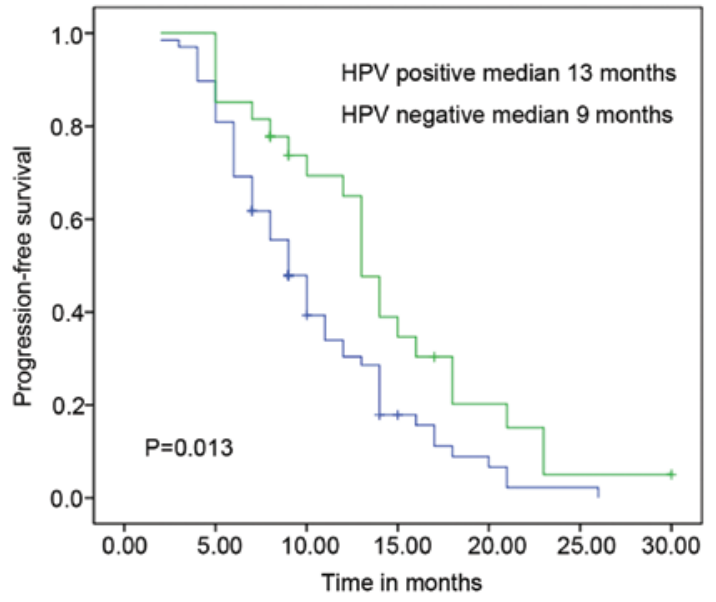

Figure 1. Progression-free survival curves according to HPV infection for advanced lung adenocarcinoma patients. HPV, human papillomavirus.

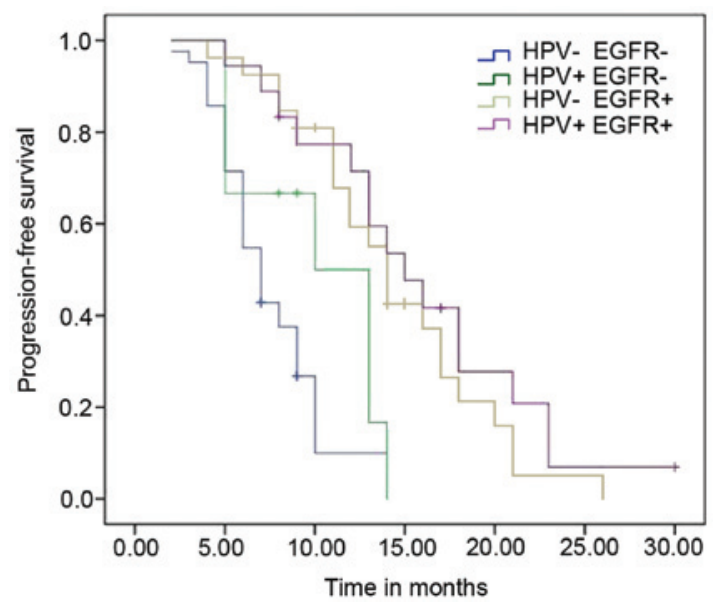

Figure 2. Progression-free survival curves for the joint assessment of HPV infection and EGFR mutation in advanced lung adenocarcinoma patients. HPV, human papillomavirus; EGFR, epidermal growth factor receptor.

revealed mean frequencies of 17 and $15 \%$ in Europe and the United States, respectively, with a higher frequency in Asia (35.7\%) (16). The most common high-risk HPV types 
Table V. HRs for PFS according to subgroups.

\begin{tabular}{|c|c|c|c|c|c|c|}
\hline HPV/EGFR mutant & Patients, $\mathrm{n}$ & $\begin{array}{l}\text { Median PFS } \\
\text { time, months }\end{array}$ & Unadjusted HR (95\% CI) & $\mathrm{P}$-value & Adjusted HR ${ }^{\mathrm{a}}(95 \% \mathrm{CI})$ & P-value \\
\hline $\mathrm{HPV}^{+}$mutant $^{+}$ & 18 & 15 & $0.581(0.445-0.758)$ & $<0.001$ & $0.640(0.488-0.840)$ & 0.001 \\
\hline $\mathrm{HPV}^{+}$mutant $^{-}$ & 9 & 10 & $0.763(0.507-1.148)$ & 0.194 & $0.829(0.538-1.275)$ & 0.393 \\
\hline $\mathrm{HPV}^{-}$mutant $^{+}$ & 26 & 14 & $0.216(0.113-0.416)$ & $<0.001$ & $0.224(0.117-0.428)$ & $<0.001$ \\
\hline $\mathrm{HPV}^{-}$mutant ${ }^{-}$ & 42 & 7 & 1.0 & & 1.0 & \\
\hline
\end{tabular}

${ }^{a}$ Adjusted hazard ratio for smoking and lymph node metastasis. HR, hazard ratio; PFS, progression-free survival; HPV, human papillomavirus; EGFR, epidermal growth factor receptor; CI, confidence interval.

identified in lung cancer patients are HPV16 and HPV18, however, other HPV types such as HPV6, 11, 31, 33, 39, 52, 58 and 82 have also been reported (17). In accordance with these reports, in the present study HPV16 was identified as the most common genotype and positivity for HPV18, 33, 58, 6 and 11 was also observed (data not shown). The overall frequency of HPV DNA in advanced NSCLC patients was $25.2 \%$ (data not shown). A previous study by Wang et al (18) demonstrated that HPV infections were present in localized and earlier stages of lung cancer, however, in the present study HPV infections were more commonly identified in patients with lymph node metastasis. The reason for these discrepancies remains unclear. We hypothesize that HPV infection may be associated with certain disease stages and metastasis in lung cancer.

Recently, NSCLC in never-smokers has emerged as a global public health issue. The cause remains unclear and few studies have focused on the prevalence of HPV in this population (19). A previous study revealed that Taiwanese never-smokers exhibited a high prevalence of HPV16/18 infection, indicating that this is a potential etiological agent of lung cancer (20). In accordance with these results, in the present study, HPV infections were observed in never-smokers (data not shown).

EGFR mutations have been demonstrated to be more common in never-smokers, women, individuals of Asian ethnicity and adenocarcinoma patients $(21,22)$. Furthermore, lung adenocarcinoma patients harboring EGFR mutations are considered to exhibit an increased sensitivity to $E G F R$ TKIs, such as gefitinib and erlotinib (23). Notably, these clinicopathological features of EGFR mutations were evident in the present study and a significant association was identified between HPV infections and EGFR mutations in advanced lung adenocarcinoma patients. However, the present study also indicated that patients with EGFR mutations exhibited a better prognosis compared with those exhibiting wild-type EGFR, regardless of HPV status, and those patients exhibited the longest PFS times, which may be due to good response to TKI- or platinum-based-adjuvant therapy. However, these findings contradict previous studies. For example, Hsu et al (24) reported survival benefits for stage I NSCLC patients that expressed the HPV16/18 E6 oncoprotein. Furthermore, Wang et al (18) demonstrated that patients with HPV infections exhibited a better survival than those without in a subgroup receiving platinum-based chemotherapy; however, this trend was not observed in subgroups receiving TKI and radiation. We hypothesize that this difference may be related to the survival index using PFS but not overall survival, and the fact that the majority of patients with EGFR mutations received TKI therapy but not platinum-based chemotherapy.

Multivariate analysis in the present study revealed that HPV DNA was significantly associated with EGFR mutations in advanced lung adenocarcinoma. Furthermore, patients with both EGFR mutations and HPV infections exhibited the longest PFS times. Márquez-Medina et al (25) previously reported that HPV infections were more common in patients harboring EGFR mutations or those sensitive to erlotinib. In addition, Wu et al (26), Zhang et al (27) and Sung et al (28) demonstrated that HPV16 E6 upregulates cellular inhibitor of apoptosis 2 (cIAP2), which is located downstream of EGFR signaling, via the EGFR/phosphatidylinositide 3-kinase (PI3K)/protein kinase B (AKT) cascade, and that cIAP2 expression was positively correlated with EGFR mutations. Therefore, the EGFR/PI3K/AKT cascade may exhibit a pivotal function in HPV-associated lung carcinogenesis in individuals with EGFR mutations.

In conclusion, the present study indicates a significant association between HPV infections and EGFR mutations in advanced lung adenocarcinoma patients. However, further large-scale prospective studies are required to confirm this hypothesis. Currently, the authors of the present study are planning a basic study to elucidate the association between HPV infections and EGFR mutations using patient-derived tumor xenograft models, which may increase current understanding with regard to the pathogenesis of HPV infections in lung cancer.

\section{Acknowledgements}

This study was supported by the National Natural Science Foundation of China (grant no. 81172172). The authors would like to thank Mr. Guo Zhen-Li (Department of Pathology, Affiliated Provincial Hospital of Anhui Medical University, Hefei, China), for collecting tissue samples and Mr. Wang $\mathrm{Xu}$ (Science and Education Division, Anhui Provincial Children's Hospital, Hefei, China) for statistical analysis.

\section{References}

1. Jemal A, Bray F, Center MM, Ferlay J, Ward E and Forman D: Global cancer statistics. CA Cancer J Clin 61: 69-90, 2011. 
2. Thun MJ, Hannan LM, Adams-Campbell LL, Boffetta P, Buring JE, Feskanich D, Flanders WD, Jee SH, Katanoda K, Kolonel LN, et al: Lung cancer occurrence in never-smokers: An analysis of 13 cohorts and 22 cancer registry studies. PLoS Med 5: e185, 2008.

3. Klein F, Amin Kotb WF and Petersen I: Incidence of human papilloma virus in lung cancer. Lung Cancer 65: 13-18, 2009.

4. Yanagawa N, Wang A, Kohler D, Santos Gda C, Sykes J, Xu J, Pintilie M and Tsao MS: Human papilloma virus genome is rare in North American non-small cell lung carcinoma patients. Lung Cancer 79: 215-220, 2013.

5. Sagerup CM, Nymoen DA, Halvorsen AR, Lund-Iversen M, Helland A and Brustugun OT: Human papilloma virus detection and typing in 334 lung cancer patients. Acta Oncol 53: 952-957, 2014.

6. Sarchianaki E, Derdas SP, Ntaoukakis M, Vakonaki E, Lagoudaki ED, Lasithiotaki I, Sarchianaki A, Koutsopoulos A, Symvoulakis EK, Spandidos DA, et al: Detection and genotype analysis of human papillomavirus in non-small cell lung cancer patients. Tumour Biol 35: 3203-3209, 2014.

7. Anantharaman D, Gheit T, Waterboer T, Halec G, Carreira C, Abedi-Ardekani B, McKay-Chopin S, Zaridze D, Mukeria A Szeszenia-Dabrowska N, et al: No causal association identified for human papillomavirus infections in lung cancer. Cancer Res 74: 3525-3534, 2014.

8. Simen-Kapeu A, Surcel HM, Koskela P, Pukkala E and Lehtinen M: Lack of association between human papillomavirus type 16 and 18 infections and female lung cancer. Cancer Epidemiol Biomarkers Prev 19: 1879-1881, 2010

9. Hasegawa Y, Ando M, Kubo A, Isa S, Yamamoto S, Tsujino K, Kurata T, Ou SH, Takada M and Kawaguchi T: Human papilloma virus in non-small cell lung cancer in never smokers: A systematic review of the literature. Lung Cancer 83: 8-13, 2014.

10. Baba M, Castillo A, Koriyama C, Yanagi M, Matsumoto H, Natsugoe S, Shuyama KY, Khan N,Higashi M, Itoh T, et al: Human papillomavirus is frequently detected in gefitinib-responsive lung adenocarcinomas. Oncol Rep 23: 1085-1092, 2010.

11. Tung MC, Wu HH, Cheng YW, Wang L, Chen CY, Yeh SD, $\mathrm{Wu}$ TC and Lee $\mathrm{H}$ : Association of epidermal growth factor receptor mutations with human papillomavirus 16/18 E6 oncoprotein expression in non-small cell lung cancer. Cancer 119: 3367-3376, 2013

12. Kato T, Koriyama C, Khan N, Samukawa T, Yanagi M, Hamada T, Yokomakura N, Otsuka T, Inoue H, Sato M, et al: EGFR mutations and human papillomavirus in lung cancer. Lung Cancer 78: 144-147, 2012.

13. Wood DE, Kazerooni E, Baum SL, Dransfield MT, Eapen GA, Ettinger DS, Hou L, Jackman DM, Klippenstein D, Kumar R, et al: Lung cancer screening, version 1.2015: featured updates to the NCCN guidelines. J Natl Compr Canc Netw 13: 23-34, 2015.

14. Wei W, Shi Q, Guo F, Zhang BY, Chen C, Zhang NS and Dong XP: The distribution of human papillomavirus in tissues from patients with head and neck squamous cell carcinoma. Oncol Rep 28: 1750-1756, 2012.
15. Zhou Q, Zhang XC, Chen ZH, Yin XL, Yang JJ, Xu CR, Yan HH, Chen HJ, Su J, Zhong WZ, et al: Relative abundance of EGFR mutations predicts benefit from gefitinib treatment for advanced non-small-cell lung cancer. J Clin Oncol 29: 3316-3321, 2011.

16. Syrjänen K: Detection of human papillomavirus in lung cancer: Systematic review and meta-analysis. Anticancer Res 32: 3235-3250, 2012.

17. Ragin C, Obikoya-Malomo M, Kim S, Chen Z, Flores-Obando R, Gibbs D, Koriyama C, Aguayo F, Koshiol J, Caporaso NE, et al: HPV-associated lung cancers: An international pooled analysis. Carcinogenesis 35: 1267-1275, 2014.

18. Wang JL, Fang CL, Wang M, Yu MC, Bai KJ, Lu PC and Liu HE: Human papillomavirus infections as a marker to predict overall survival in lung adenocarcinoma. Int J Cancer 134: 65-71, 2014.

19. Cheng YW, Chiou HL, Sheu GT, Hsieh LL, Chen JT, Chen CY, $\mathrm{Su}$ JM and Lee H: The association of human papillomavirus 16/18 infection with lung cancer among nonsmoking Taiwanese women. Cancer Res 61: 2799-2803, 2001.

20. Pallis AG and Syrigos KN: Lung cancer in never smokers: Disease characteristics and risk factors. Crit Rev Oncol Hematol 88 494-503, 2013.

21. Mitsudomi T, Kosaka T and Yatabe Y: Biological and clinical implications of EGFR mutations in lung cancer. Int J Clin Oncol 11: 190-198, 2006.

22. Shigematsu H, Lin L, Takahashi T, Nomura M, Suzuki M, Wistuba II, Fong KM, Lee H, Toyooka S, Shimizu N, et al: Clinical and biological features associated with epidermal growth factor receptor gene mutations in lung cancers. J Natl Cancer Inst 97: 339-346, 2005.

23. Paez JG, Janne PA, Lee JC, Tracy S, Greulich H, Gabriel S, Herman P, Kaye FJ, Lindeman N, Boggon TJ, et al: EGFR mutations in lung cancer: Correlation with clinical response to gefitinib therapy. Science 304: 1497-1500, 2004

24. Hsu NY, Cheng YW, Chan IP, Ho HC, Chen CY, Hsu CP, Lin MH and Chou MC: Association between expression of human papillomavirus 16/18 E6 oncoprotein and survival in patients with stage I non-small cell lung cancer. Oncol Rep 21: 81-87, 2009.

25. Márquez-Medina D, Gasol-Cudós A, Taberner-Bonastre MT, Samamé Pérez-Vargas JC, Salud-Salvia A and Llombart-Cussac A: Human papillomavirus in non-small-cell lung cancer: The impact of EGFR mutations and the response to erlotinib. Arch Bronconeumol 49: 79-81, 2013 (In English, Spanish).

26. Wu HH, Wu JY, Cheng YW, Chen CY, Lee MC, Goan YG and Lee H: cIAP2 upregulated by E6 oncoprotein via epidermal growth factor receptor/phosphatidylinositol 3-kinase/AKT pathway confers resistance to cisplatin in human papillomavirus 16/18-infected lung cancer. Clin Cancer Res 16: 5200-5210, 2010.

27. Zhang E, Feng X, Liu F, Zhang P, Liang J and Tang X: Roles of PI3K/Akt and c-Jun signaling pathways in human papillomavirus type 16 oncoprotein-induced HIF-1 $\alpha$, VEGF, and IL-8 expression and in vitro angiogenesis in non-small cell lung cancer cells. PLoS One 9: e103440, 2014.

28. Sung WW and Lee $H$ : The role of interleukin-10 in the progression of human papillomavirus-associated lung carcinoma. Oncoimmunology 2: e25854, 2013. 